Special Issue of the 8th International Advances in Applied Physics and Materials Science Congress (APMAS 2018)

\title{
Space Charge Lenses for Intensive Ion Beams Formation
}

\author{
I.S. HASAnOV ${ }^{a, *}$, V.A. Orudjev ${ }^{b}$, I.I. Gurbanov ${ }^{a}$ AND E.M. AkBArov ${ }^{a}$ \\ ${ }^{a}$ Institute of Physics of ANAS, G. Javid ave., 131, Az1143, Baki, Azerbaijan \\ ${ }^{b}$ BP Caspian Exploration Ltd, 153, Neftchiler Ave, Az1010, Baki, Azerbaijan
}

\begin{abstract}
The space charge lenses intended for focusing of ion beams have been proposed for the first time by D. Gabor in 1947. Their optical force exceeds on 2 orders of magnitude optical force of electrostatic and on 4 orders optical force of magnet lenses with other things being equal. The basic advantage of a Gabor lens, besides high optical force, consists in ability to focus intensive ion beams as an ion space charge does not deform initial distribution of potential in a lens. The distribution of focusing potential is created by a space charge of compensating electrons. The theoretical and experimental research of Gabor lenses has led to creation of the plasma optics - a new section of plasma physics. These lenses are successfully applied to focusing the high current and of high energy ion beams, and beams of heavy ions also. The use of space charge lenses is of interest for additional heating of thermonuclear plasma in installations TOKAMAK at injection of intensive ion beams with their subsequent neutralization.
\end{abstract}

DOI: 10.12693/APhysPolA.135.841

PACS/topics: ion beam, Gabor lens, plasma optics, focus, aberration

\section{Introduction}

Classical electrostatic and magnetic lenses exhibit rather low optical force due to the presence in them focusing and defocusing areas. Such a lenses carry out focusing of the second order [1]. Besides, electrostatic lenses are not suitable for focusing intensive ion beams as the space charge of ions deforms essentially vacuum distribution of potential in a lens. Focusing beams of heavy ions by means of magnet lenses is also inconvenient, because of the dependence of optical force on ion mass.

The specified difficulties have been overcome by means of space charge lenses. The design of such a lens for focusing beams of positive ions has been proposed for the first time by D. Gabor in 1947 [2]. It consists of the electromagnetic trap for thermal electrons and a system of the cylindrical electrodes symmetrically located in a non-uniform magnetic field. The intensity of a magnetic field is matched to limit the cross-section of electrons diffusion, that is it does not influence ions. Thus, a Gabor lens is the electrostatic lens to an essence. The volume of a lens is filled by electrons; an electric field is focusing everywhere; therefore the lens carries out focusing of the first order.

Characteristics of space charge lens are considered within the framework of the new section of plasma physics - plasma optics which have been created on the basis of the theoretical analysis of electron component properties in the hydrodynamic approach [3]. The main principle of the plasma optics is an equipotentiality principle of magnetic force lines in cold plasma. Due to the high mobility of electrons along a magnet field and

*corresponding author; e-mail: ilkhamg@yahoo.com essentially limited mobility across a field, the electric field crossed with magnetic is realized in similar to a plasma environment

$$
\boldsymbol{E}+\frac{1}{c}\left[\boldsymbol{v}_{e}, \boldsymbol{H}\right]=0,
$$

where $\boldsymbol{v}_{e}$ is the drift speed of the electrons.

By changing the configuration of electric (and magnet) fields, it is possible to create various plasma devices: plasma (Hall) accelerators, electrostatic traps, plasma lenses, energy analyzers, recuperate. The formula for a focal length of a Gabor lens, which the inventor named as plasma lens, has been determined as

$$
f=\frac{R W}{2 \theta e U_{l}}
$$

where $R$ is the radius of a lens, $W$ - energy of ions, $\theta$ - dimensionless parameter close to unity, $e$ - an electron charge, $U_{l}$ - a voltage of a lens. This formula was deduced in the assumption, that $e U_{l} \ll W$. The analysis shows that the optical force of a plasma lens is on 2 orders of magnitude more than the optical force of an electrostatic lens and on 4 orders more than the optical force of a magnet lens with other things being equal. The focal length of a Gabor lens does not depend on a specific charge of particles, it can be scattering also. The intensive ion beam does not distort the distribution of potential in a lens, as its space charge always is overcompensated by a charge of captured electrons.

\section{Experiment}

Our experiments were carried out on the vacuum installation which schema is presented on Fig. 1. Beams of gas ions ( $\mathrm{He}, \mathrm{N}_{2}$, Ar) were obtained by means of the duoplasmatron 1 . The magnet field in a plasma lens was created with the help of the coil 3 with an adjustable current. Electrodes of a lens represent 9 coaxially - located rings of stainless steel, the internal diameter of ring is $26 \mathrm{~mm}$, the extent diameter of the system of electrodes 
— $76 \mathrm{~mm}$. The tubular compensator 6 serves for input of electrons in the volume of a lens as a result of ionelectron emission from its internal surface. By means of the probe 7 is measured the distribution of potential in a lens and by means of the diaphragm 5 controls the radial distribution of an ion current density. The total current of a beam is measured with the help of a collector 4. Duoplasmatron has been operated both in permanent and in pulse regimes.

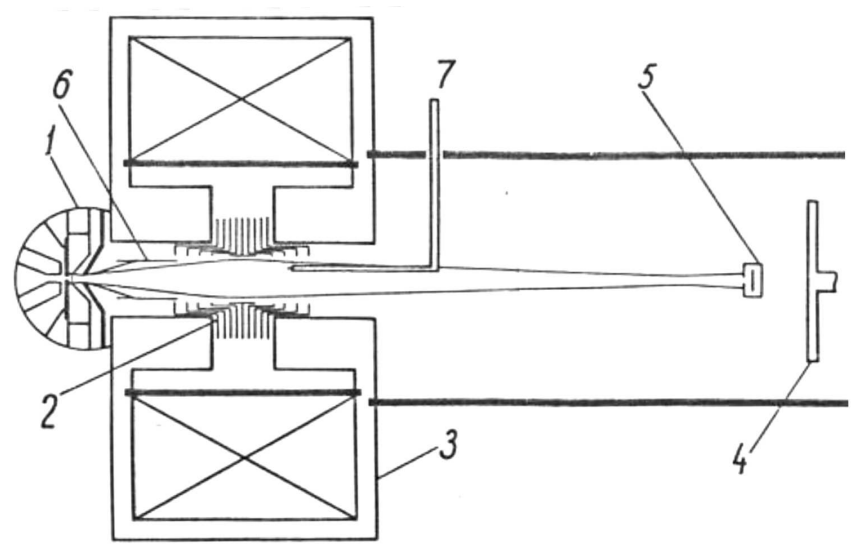

Fig. 1. Schema of experimental installation [7]: 1 extraction system of duoplasmatron; 2 - electrodes; 3 - magnet coil; 4 - collector; 5 - diaphragm; 6 compensator; 7 - probe.

\section{Results and discussion}

The experiments executed for a number of years have confirmed a prediction of the theory and have revealed specific features of space charge lenses. Depending on the concentration of an ion beam, an environment in a lens can be close to quasi-neutral or can be close to electron gas. The high optical force of such lenses has been confirmed according to the formula (1), including, at focusing megavoltage ion beams [4-6]. The self-consistent regime of a lens action without submission of an electric voltage on isolated electrodes [5] was observed. They were charged in this case as a result of the hit on them a peripheral part of a beam of helium ions. Thus, the lens without an electric feed can be created: the electric field is formed due to charging electrodes by ions, and the magnetic field is created by a permanent ring magnet.

At focusing pulse ion beams the phenomena caused by dynamics of an electric field formation in a volume of a lens are found out. When electrons compensating a space charge of the beam are formed by beams as a result of ion-electron emission of the compensator (Fig. 1), filling a lens with electrons needs a certain time. In our experiments the filling time reaches to tens of microseconds [7], therefore the front of a pulse of a current density in focus appreciably lagged behind the front of a pulse of the total current of the beam (Fig. 2). The increasing time of the total current is determined by an ion source - the duoplasmatron. In the pulse self-consistent mode the moment of achievement of the maximal potential on the central electrode precisely coincide with the moment of the achievement of the maximal current density of a beam in focus (Fig. 3), that is focusing of a beam occurs after formation of an electric field in the lens volume.

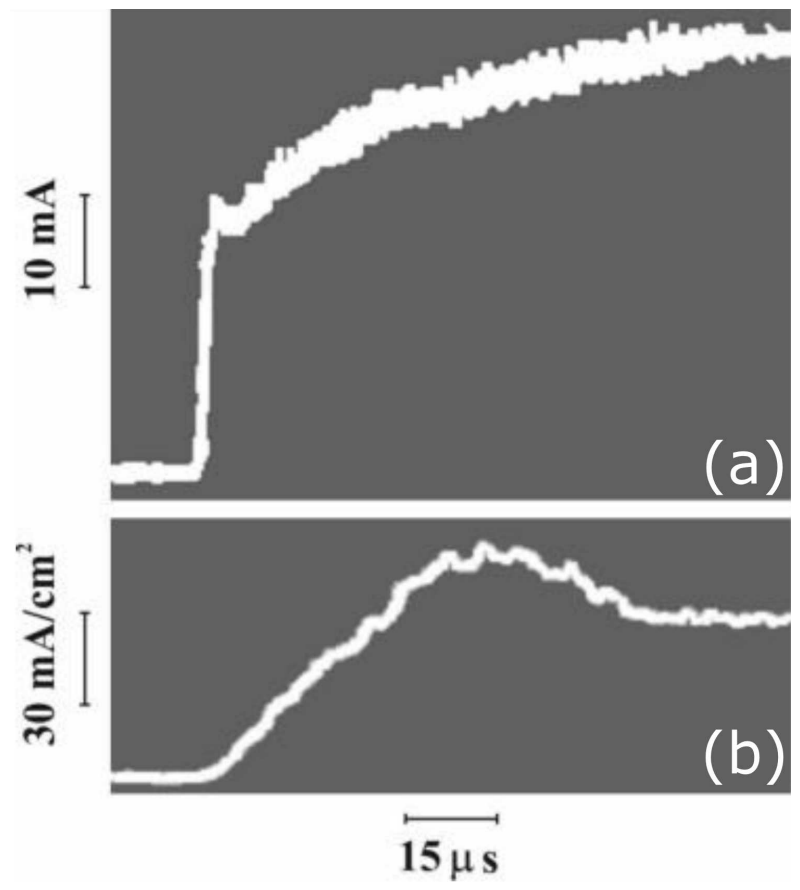

Fig. 2. Oscillograms of beam current on the collector (a) and of current density in crossover (b). $W=25 \mathrm{keV}$, $p=6 \times 10^{-6}$ Torr, $U_{l}=2.9 \mathrm{kV}, H=1 \mathrm{kOe}$.

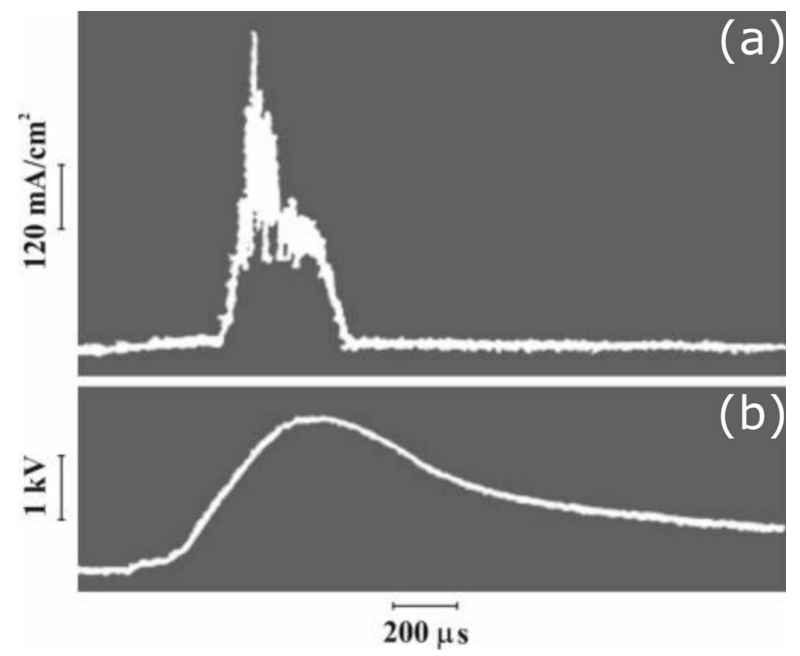

Fig. 3. Oscillograms of current density in crossover (a) and potential of central electrode $(\mathrm{b})$ at self-consistent mode. $W=28 \mathrm{keV}, I=30 \mathrm{~mA}, H=1 \mathrm{kOe}, p=$ $7 \times 10^{-6}$ Torr. 
Due to electrons drift rotation in the crossed electromagnetic fields, fluctuations of space potential in Gabor lens grow. At low pressure of the residual gas, the highfrequency diocotron instability of an electron cloud develops because of the relative movement of the adjacent layers with different radia [8]. At a nonlinear stage of instability, relative move of layers stops and the cloud rotates as a rigid rotator. It corresponds to the constant concentration of electrons and square-law dependence of potential on the radius that corresponds to ideal optics (see below). At the certain pressure of residual gas it is ionized by the ion beam; secondary slow ions are underlied to radial oscillations in a potential well of a lens, and their concentration near to an axis of system prevails. Oscillations of slow ions can appear in resonance with the rotation of an electron cloud, intensive ion-electron Buneman instability [9] arises in this case. It has rather low frequency and is suppressed at infringement of resonance (Fig. 4). Fluctuations in a plasma lens worsen the quality of focusing and reduce an ion current density in a crossover of a beam [10].

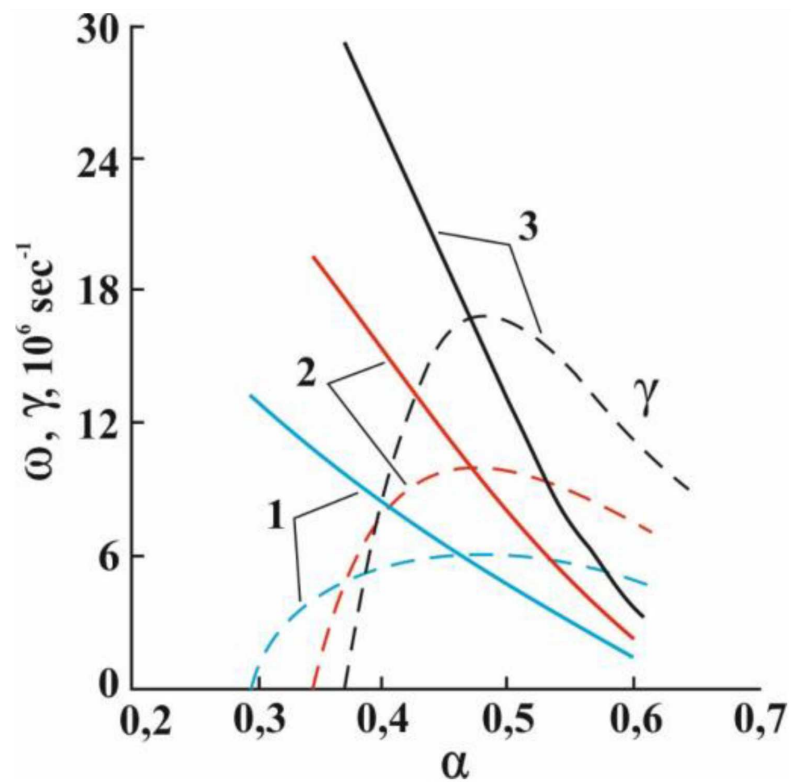

Fig. 4. Calculated dependence of frequency $\omega$ and increment $\gamma$ of electron-ion oscillations from parameter $\alpha=n_{i} / n_{e}$, working gas $\mathrm{N}_{2}, H=1 \mathrm{kOe}, 1-U_{l}=1 \mathrm{kV}$, $2-U_{l}=2 \mathrm{kV}, 3-U_{l}=3 \mathrm{kV}$.

Updating Gabor lenses were investigated and applied to transportation and focusing intensive beams of heavy ions $[11,12]$. It is of interest in connection with the problem of thermonuclear synthesis and a number of technological tasks. The opportunity of the creation of a space charge lens for focusing beams of negative ions has been shown also. In this case, the cloud of a positive space charge owing to ionization of residual gas by fast negative ions in a lens is formed, and formed electrons leave the area of a lens as the negative potential is applied on the central electrode [13].
The important characteristics of optical system are its aberrations which determine the quality of beam focusing. As the experiments have shown, the most dangerous distorsion of a plasma lens is the spherical aberration which limits the current density of the focused beam [10]. One of the reasons of an aberration is an accumulation of secondary ions in the areas close to the axis of a lens that causes enough high potential of space in this zone. Dependence of the intensity of an electric field on radius becomes nonlinear, and in the result, the peripheral area of a beam is focused stronger, than near-axis zone, and the focus of a beam is bluring. A reduction in the level of aberrations of Gabor lens was considered in numerous publications. In particular, the lens with the minimized gradient of a magnetic field which has allowed reducing in the certain degree a spherical aberration and emittance of a beam was used [14].

\section{Conclusion}

Now we carry out researches on the elimination of a spherical aberration of a plasma lens which reason is a number of factors: the high potential of near axis area, the non-optimal arrangement of electrodes relative to magnetic field, the non-optimal distribution of potential on electrodes. Experiments are conducted in two directions: (a) the lens is filled by electrons by means of the beam (ion-electron emission of the compensator) and (b) the lens is filled by electrons by means of the thermoelectron emitter. In the first case, is used the duoplasmatron as an ion source (Ar, $\mathrm{He}$ ), and the formation of an electric field in a lens needs a certain time. In the second case, the liquid metal source serves as an ion source ( $\mathrm{Sn}, \mathrm{In}$, $\mathrm{Au}$ ), and the electric field in a lens is formed irrespective of a beam. The purpose of reduction in a spherical aberration is obtaining of sharply focused beams for surface structures formation.

\section{References}

[1] M. Szilagyi, Electron and ion optics, Plenum Press, New York 1988 p. 515.

[2] D. Gabor, Nature 160, 89 (1947).

[3] A.I. Morozov, Introduction to plasma dynamics, PHYSMATLIT, Moscow 2006.

[4] V.V. Jukov, A.I. Morozov, G.Ya. Shepkin, Lett. J. Exper. Theor. Phys. 9, 24 (1969).

[5] M.D. Gabovich, I.S. Hasanov, I.M. Protsenko, Lett. J. Exper. Theor. Phys. 3, 1153 (1977).

[6] R. Booth, H.W. Lefevre, Nucl. Instr. Meth. 151, 3112 (1978).

[7] I.S. Hasanov, I.M. Protsenko J. Tech. Phys. 52, 1783 (1982).

[8] I.S. Hasanov, I.M. Protsenko Lett. J. Tech. Phys. 9, 1228 (1983).

[9] R.H. Levy, J.D. Daugherty, O. Buneman, Phys. Fluids 12, 2616 (1969). 
[10] I.S. Hasanov, A.A. Goncharov, I.M. Protsenko, Plasma optical systems of ion beams formation and focusing, Preprint Ins. Phys. AS USSR, Kiev 1990, p. 39 .

[11] A.A. Goncharov, I.M. Protsenko, I.G. Brown, Rev. Sci. Instr. 69, 1135 (1998).

[12] A.A. Goncharov, Rev. Sci. Instr. 84, 021101 (2013).
[13] A.A. Goncharov, A.M. Dobrovolskiy, S.M. Dunets, I.V. Litovko, V.I. Gushenets, E.M. Oks, Rev. Sci Instr. 83, 02B723 (2012).

[14] A.A. Goncharov, I. Brown, IEEE Trans. Plasma. Sci. 35, 986 (2007). 